\title{
PEGylated Nanoemulsions Containing 1,2- Distearoyl-sn-Glycero-3-Phosphoglycerol Induced Weakened Accelerated Blood Clearance Phenomenon
}

\section{Zhe Li}

Fourth Affiliated Hospital of China Medical University

\section{Xin Gao}

Shenyang Pharmaceutical University

\section{Xinyang Yan}

Shenyang Pharmaceutical University

\section{Yuihui Deng}

Shenyang Pharmaceutical University

Haiying Ma ( $\square$ cmu4h-mhy@126.com )

Fourth Affiliated Hospital of China Medical University

\section{Research Article}

Keywords: PEGylated emulsions, 1,2-distearoyl-sn-glycero-3-phosphoglycerol, accelerated blood clearance phenomenon

Posted Date: October 21st, 2021

DOl: https://doi.org/10.21203/rs.3.rs-911131/v1

License: (c) (1) This work is licensed under a Creative Commons Attribution 4.0 International License. Read Full License

Version of Record: A version of this preprint was published at Drug Delivery and Translational Research on January 29th, 2022. See the published version at https://doi.org/10.1007/s13346-021-01111-4. 


\section{Abstract}

Injections of polyethylene glycol (PEG)-modified nanomedicines can lead to an accelerated clearance of the next dose of PEGylated nanomedicines, which is referred to as the accelerated blood clearance (ABC) phenomenon. It has been reported that anti-PEG IgM plays an important role in the induction of the $A B C$ phenomenon, identifying the interface between the main chain of PEG and the hydrophobic segment of the repeated injections of the PEGylated nanocarriers, resulting in increased liver uptake and loss of long cycle characteristics. In this study, we demonstrated that the 1,2-distearoyl-sn-glycero-3-phosphoglycerol (DSPG) in PEGylated nanoemulsions (PEs) may mask this interface between the main chain of PEG and the hydrophobic segment, inhibiting the recognition and binding of anti-PEG IgM to PEs, and evidently weakening the $A B C$ phenomenon of PEs. This will provide a novel strategy to improve the curative effect of PEGylated nanocarriers.

\section{Introduction}

Polyethylene glycol (PEG) is used widely with protein and nanocarriers in the pharmaceutical field because of its good water solubility and biocompatibility. PEGylation refers to the process of conjugating PEG to drugs or nanocarriers by chemical means $[1,2]$. Use of this technology has endowed the modified drugs or nanocarriers with better hydrophilic properties, thereby lowering their immunogenicity and improving their stability; therefore, its use has been widely favored by researchers. As early as 1977, researchers developed the technique of attaching PEG to the surface of proteins, which remarkably prolonged the circulation time of proteins and reduced their immunogenicity [3, 4]. In 1990, the first PEGylated protein product approved by the FDA, Adagen ${ }^{\circledR}$, a PEGylated adenosine deaminase, was used to treat severe immunodeficiency diseases [5]. However, since the first PEGylated nanomedicine, Doxil $\circledast$, was approved for commercial distribution in 1995, there have been no other approved high-density PEGylated nanocarriers, indicating a stagnation in the development of PEGylated nanomedicine [6-9].

Several clinical and experimental studies have revealed that systemic injection of PEGylated nanomedicines such as PEGylated liposomes [10], proteins[11], micelles [12,13], emulsions[14, 15], and nanoparticles [16] may elicit an anti-PEG antibody (APA) response, and that repeated administration may result in the accelerated blood clearance (ABC) phenomenon. Repeated intravenous injections of complex nano-drugs, such as PEGylated protein or nanoparticles, in animals or humans showed that with the second and subsequent doses the circulation time was shortened sharply and the curative effect reduced.

IgM produced by PEGylated nanomedicines may cause serious problems in the field of drug targeting. After a single subcutaneous or intravenous injection of the PEGylated recombinant mammalian uricase, Krystexxa, approved by the FDA in 2010, 38\% of patients experienced an APA response; when injected repeatedly, even if the intervals between the two administrations was one year, the APA response was quick, resulting in failure of the therapy [17]. Non-cytotoxic anticancer drugs (such as genes) and diagnostic drugs loaded with PEGylated nanocarriers can lose their ability to treat or diagnose, due to repeated injections. Nowadays, "treatment" involves the use of imaging technology where PEGylated 
nanomedicines are often used as diagnostic and therapeutic carriers. However, the efficiency of the second injection may be seriously affected when the immune response is activated by the PEGylated nanomedicines of the first dose, that is, the diagnostic system. Therefore, the ABC phenomenon must be avoided as it can influence the reliability of the diagnosis. The occurrence of the $A B C$ phenomenon leaves only one chance for treatment and diagnosis with nanocarriers. However, this limitation must be overcome in clinical settings for successful diagnosis and treatment. Studies have shown that the immunogenicity of PEGylated nanomedicines poses a threat to clinical patients [7, 18-20], and that the change in pharmacokinetic behavior damages the advantages of PEGylation. Thus, the ABC phenomenon poses an unavoidable challenge to further research and the application of PEGylation technology.

Various strategies have been developed to attenuate or abolish the $A B C$ phenomenon $[11,21,22)$, including the pre-injection of high-molecular-weight PEG [23] or anti-PEG scFv, the regulation of the physical and chemical properties of PEGylated nanomedicines [14, 24], adjusting the administration regimens[25,26], and replacing it with other polymers or co-modifications [27-29]. For materials such as $\mathrm{mPEG}_{2000}-\mathrm{DSPE}$, the junction (amide bond) between the hydrophilic PEG chain and hydrophobic DSPE fragment may be the key site for the recognition and mediation of the scavenging of PEGylated nanomedicines by anti-PEG IgM [30]. In this study, we used 1,2-distearoyl-sn-glycero-3-phosphoglycerol (DSPG) and prepared a series of PEs containing DSPG to investigate the ability of DSPG to inhibit the $A B C$ phenomenon in Wistar rats.

\section{Materials And Methods \\ 2.1. Materials}

1,1'-Dioctadecyl-3,3,3',3'-tetramethylindotricarbocyanine iodide (DiR) was purchased from AAT Bioquest, Inc. (USA). DSPG, and soybean phosphatidylcholine S75 (S75) were obtained from Lipoid GmbH (USA). N (carbonyl-methoxy PEG 2000 )-1,2-distearoyl-sn-glycero-3-phosphoethanolamine ( $\mathrm{mPEG}_{2000}$-DSPE) was obtained from Genzyme Corporation (USA). Medium-chain triglycerides (MCTs) were purchased from Beiya Medicated Oil Co., Ltd. (China). Bovine serum albumin (BSA) was supplied by Shanghai Seebio Biotech, Inc. (China). Horseradish peroxidase (HRP)-conjugated goat anti-rat IgM antibody was obtained from ICL Lab (USA). The compound 1,2-diaminobenzene was purchased from Sigma-Aldrich (USA). All the other reagents were of analytical grade and purchased commercially.

\subsection{Animals}

Male Wistar rats (180-200 g) were purchased from the Experimental Animal Center of the Shenyang Pharmaceutical University (Shenyang, China). All the animal experiments were conducted according to guidelines of the animal welfare committee of Shenyang Pharmaceutical University (NIH publication \#85-23, revised in 1985).

\subsection{Preparation of PEs}


The different formulations of the PEs used are shown in Table 1. The different PEs were prepared using the DiR as the marker at a concentration of $1.8 \mathrm{mg} / \mathrm{mL}$ with the oil phase MCT prescription amount being $30 \mathrm{mg} / \mathrm{mL}$, and the lipid prescription composition as shown in Table 1. All the PEs were prepared according to the following method. The S75, $\mathrm{mPEG}_{2000}$-DSPE, MCT, and DiR (Table 1) was mixed in sterile water and heated to $55^{\circ} \mathrm{C}$. Sterile water was added to the oil phase at the same temperature and stirred quickly. DSPG at $\mathrm{n}$ mol\% PE-DSPGs without any S75 was diluted in sterilized water. The mixture was stirred at $55^{\circ} \mathrm{C}$ for 20 min and sonicated in an ice bath for 8 minutes using a laboratory ultrasonic cell pulverize to prepare the emulsions. Then, nanoemulsions were prepared by extrusion through 0.8 -, 0.45 , and $0.22-\mu \mathrm{m}$ polycarbonate membranes. Using different PEG modification densities, $\mathrm{n}$ mol \% PE and $n$ mol \% PE-DSPG $(n=10,30,50)$ were prepared with $5 \mu$ mol phospholipid $/ \mathrm{mL}$. Different DSPG concentrations, PE-DSPG- $n(n=2,5,9,15,30)$ with a fixed $\mathrm{mPEG}_{2000}$-DSPE concentration of 0.5 $\mu \mathrm{mol} / \mathrm{mL}$, were prepared. The distribution of the particle sizes and zeta potentials of the nanoemulsions were determined using a Nicomp 380. The entire process was performed without exposure to light.

Table 1

Formulations of PEs.

\begin{tabular}{|c|c|}
\hline Formula & $\begin{array}{l}\text { Lipid composition } \\
(n / n)\end{array}$ \\
\hline $10,30,50 \mathrm{~mol} \% \mathrm{PE}$ & S75: $\mathrm{mPEG}_{2000^{-D S P E}(9: 1,7: 3,5: 5)}$ \\
\hline 10, 30, $50 \mathrm{~mol} \%$ PE-DSPG & DSPG: mPEG $_{2000}$-DSPE $(9: 1,7: 3,5: 5)$ \\
\hline PE-DSPG 2,5,9,15,30 & DSPG: $0.5 \mu \mathrm{mol} / \mathrm{ml} \mathrm{mPEG} 2000^{-D S P E}(2: 1,5: 1,9: 1,15: 1,30: 1)$ \\
\hline
\end{tabular}

\subsection{Pharmacokinetics of PEs}

The rats were divided randomly into 28 groups ( $n=3$ in each group). The injection schemes with seven days interval between the two PE injections are shown in Table 2. The rats were administered PEs intravenously at a rate of $5 \mu \mathrm{mol}$ phospholipid $/ \mathrm{kg}$. The DiR was used to demonstrate the pharmacokinetics of the nanoemulsions at $0.3 \mathrm{mg} / \mathrm{kg}$ due to the high encapsulation rate of nanoemulsions. At $0.0167,0.083,0.25,0.5,1,4$, and $8 \mathrm{~h}$ after the first and second injections, $0.5 \mathrm{~mL}$ plasma was collected from the ophthalmic venous plexus and placed in a heparinized tube. The plasma was separated and the DiR was measured as described previously (Liu et al., 2020). 
Table 2

Injection Schemes for PEs in Wistar rats $(n=3)$

\begin{tabular}{|c|c|c|}
\hline Groups & First dose & Second dose \\
\hline Single $\mathrm{n}$ mol \% PEs & $5 \%$ Glu & $10,30,50 \mathrm{~mol} \%$ PEs \\
\hline Single PE-DSPG-n & $5 \%$ Glu & PE-DSPG-2,5,9,15,30 \\
\hline $\begin{array}{l}\text { Single } \mathrm{n} \mathrm{mol} \mathrm{\%} \mathrm{PE-} \\
\text { DSPGs }\end{array}$ & $5 \%$ Glu & $10,30,50 \mathrm{~mol} \%$ PE-DSPGs \\
\hline $\begin{array}{l}\text { Repeated n mol \% } \\
\text { PEs }\end{array}$ & $\begin{array}{l}10,30,50 \mathrm{~mol} \% \\
\text { PEs }\end{array}$ & $10,30,50 \mathrm{~mol} \%$ PEs \\
\hline Repeated PE-DSPG-n & $\begin{array}{l}\text { PE-DSPG- } \\
2,5,9,15,30\end{array}$ & PE-DSPG-2,5,9,15,30 \\
\hline $\begin{array}{l}\text { Repeated n mol \% } \\
\text { PE-DSPGs }\end{array}$ & $\begin{array}{l}\text { 10,30,50 mol \% PE- } \\
\text { DSPGs }\end{array}$ & $10,30,50 \mathrm{~mol} \%$ PE-DSPGs \\
\hline Cross-administration & $10 \mathrm{~mol} \% \mathrm{PE}$ & $30 \mathrm{~mol} \%$ PE, PE-DSPG and PE-DSPG-30 \\
\hline Co-injection & $10 \mathrm{~mol} \% \mathrm{PE}$ & $\begin{array}{l}30 \mathrm{~mol} \% \mathrm{PE}, \mathrm{PE}-\mathrm{DSPG} \text { and PE-DSPG-30 with } 10 \mathrm{~mol} \\
\% \mathrm{PE} \text { respectively }\end{array}$ \\
\hline
\end{tabular}

\subsection{Determination of anti-PEG IgM in serum}

An improved enzyme-linked immunosorbent assay (ELISA) method was performed according to the classical ELISA method to determine the anti-PEG IgM in serum, as described previously [14]. Blood samples were collected and incubated at $25^{\circ} \mathrm{C}$ for $2 \mathrm{~h}$. Serum was separated by centrifugation at $800 \mathrm{~g}$ for $10 \mathrm{~min}$, and the anti-PEG IgM was determined.

\subsection{Statistics}

Comparisons between the two groups were conducted using the Student's $t$-test with SPSS software. Data in all the tables and figures were represented as the means \pm standard deviations (SDs), and values at $P<0.05$ were considered statistically significant.

\section{Results}

\subsection{Characteristics of the PEs}

The particle size of the PEs was controlled between 119 and $131.2 \mathrm{~nm}$, the polydispersity index (PDI) of each nanomedicine less than 0.2 , and the zeta potential below $-20 \mathrm{mV}$, indicating that the nanomedicines showed good stability (Table S1).

As everyone knows, the phase transition temperature of SPC is $-20^{\circ} \mathrm{C}$, while that of DSPG is $55^{\circ} \mathrm{C}$. In order to eliminate the influence of the two emulsifiers on the release of emulsions in blood, we compared the release behavior of different emulsions in vitro simulated in vivo environment. 
$1.0 \mathrm{~mL}$ PEs was precisely aspirate and added to a dialysis bag (with a molecular weight cut off of $10 \mathrm{kDa}$ ), clamped the two ends and placed it in $200 \mathrm{~mL}$ of PBS buffer $(500 \mathrm{mmol} / \mathrm{L}, \mathrm{pH}=7.4$, containing appropriate penicillin), stirred under constant temperature at $37.0 \pm 0.5^{\circ} \mathrm{C}$ and away from light (100rpm). At $0.5,1,2,4,8,12,24$, and $48 \mathrm{~h}$, drew $3.0 \mathrm{~mL}$ of dialysate, and added an equal amount of release medium. The dialysate was filtered with a $0.45 \mu \mathrm{m}$ microporous filter membrane and the fluorescence intensity was measured at $\lambda_{\mathrm{ex}}=750 \mathrm{~nm}, \lambda_{\mathrm{em}}=790 \mathrm{~nm}$, and the concentration was calculated by substituting it into the standard curve equation; the cumulative release $\mathbf{R n}$ of the drug is calculated, the formula is as follows, and the results are as follows Fig. 1.

$$
\mathbf{R n}=\frac{\mathrm{CnV} 0+\sum_{\mathbf{n}-1}^{\mathbf{n}} \mathbf{C}(\mathbf{n}-1) \mathbf{V}}{\mathrm{Mt}} \times 100 \%
$$

Among them, $\mathbf{C n}$ is the concentration at the $\mathrm{nth}$ sampling, $\mathbf{V} \mathbf{0}$ is the volume of the release medium, $\mathbf{V}$ is the volume of each sampling, and $\mathbf{M t}$ is the total drug concentration. Results showed that there was no significant difference in the release behavior among different PE.

\subsection{Effect of the PEG modified densities on the pharmacokinetics of $\mathrm{n} \mathrm{mol} \%$ PEs and PE-DSPGs of single and repeated intravenous injection}

The 8-hour pharmacokinetic behavior of a single injection of $\mathrm{n}$ mol \% PEs and PE-DSPGs was evaluated by measuring the plasma concentration of the DiR. As indicated by the area under the curve (AUC) and $T_{1 / 2}$, the circulation time of $n$ mol \% PEs and PE-DSPGs in the Wistar rats increased gradually with the increase in PEG density on the surface of $n$ mol \% PEs and PE-DSPGs (Table S2). Seven days after the पrst injection, the rats were injected repeatedly.

The strength of the $A B C$ phenomenon was evaluated using the $A B C_{\text {index }}$, which indicated the ratio of $A U C$ of the second dose to that of the first dose; that is, the $A B C_{\text {index }}=A U C_{(0-60 \mathrm{~min})}$ of the second injection $/ A U C_{(0-60 \mathrm{~min})}$ of the first injection. The higher the $A B C_{\text {index }}$ value, the smaller the difference in pharmacokinetic behavior between the second and first injections, which meant that a weaker $A B C$ phenomenon is occurring. Here, the $A B C_{\text {index }(0-60 \mathrm{~min})}$ was used as the criterion to evaluate the strength of the $A B C$ phenomenon.

The repeated administration of $\mathrm{n}$ mol \% PEs in each group induced the ABC phenomenon. When the modification density of PEG increased from $10 \mathrm{~mol} \%$ to $30 \mathrm{~mol} \%$, the $A B C$ phenomenon decreased; however, when the PEG modification density continued to increase, the ABC phenomenon was enhanced. Compared with $\mathrm{n} \mathrm{mol} \% \mathrm{PEs}$, there were no significant differences in the level of anti-PEG IgM induced by $\mathrm{n}$ mol \% PE-DSPGs with the same PEG modification density (Fig. 2), but the $A B C_{\text {index }}$ of each group was significantly higher than that of $\mathrm{n}$ mol \% PEs, with the same PEG modification density, indicating that the 
$A B C$ phenomenon induced by each group was remarkably weakened. When the $A B C_{\text {index }}$ of $30 \mathrm{~mol} \% \mathrm{PE}-$ DSPG reached 0.89 , almost no $A B C$ phenomenon was observed (Fig. 3). These results suggest that both the modification density of PEG and the addition of DSPG could affect the intensity of the ABC phenomenon. The modification density of PEG affected the secretion levels of anti-PEG IgM, whereas while DSPG did not affect the secretion level of antibodies, it weakened the ABC phenomenon.

\subsection{Effect of DSPG concentration on the pharmacokinetics of single and repeated injections of PE-DSPG-ns}

To further investigate the influence of DSPG on the ABC phenomenon, we prepared a series of PEs with different DSPG concentrations, PE-DSPG-ns, using the same procedure as that for $\mathrm{n} \mathrm{mol} \%$ PEs and PEDSPGs (Fig. 4). No significant differences were observed in the pharmacokinetic behavior among the five groups after a single injection of PE-DSPG-ns, as shown by the AUCs and $T_{1 / 2}$ values (Table S2). Seven days after the first injection, the PE-DSPE-ns containing DiR were injected repeatedly with the same phospholipid dose. The $A B C_{\text {index }}$ showed that the addition of DSPG could indeed weaken the ABC phenomenon. When the modification concentration of PEG was fixed, i.e., PE-DSPG-ns groups, the ABC phenomenon gradually weakened with the gradual increase of DSPG concentration and when the molar ratio of the DSPG to PEG increased to $30: 1$ in the PE-DSPG-30 group, the $A B C_{\text {index }}$ reached approximately 0.94 , and the $A B C$ phenomenon almost disappeared.

No significant differences were observed in the level of anti-PEG IgM induced by the first injection of PEs with the same PEG modification density; however, the addition of DSPG weakened the ABC phenomenon in each group, significantly. Therefore, we selected $5 \mu \mathrm{mol}$ phospholipid $/ \mathrm{kg} 10 \mathrm{~mol} \% \mathrm{PE}$ with the smallest $A B C_{\text {index }}$ for the first injection, and the rats with high levels of anti-PEG IgM after the first injection of 10 mol \% PE were termed as ABC (+) rats. As shown in Fig. 5(A-C), the ABC (+) rats produced a high level of anti-PEG-IgM 7 days after the first injection while the second $30 \mathrm{~mol} \% \mathrm{PE}$ injection produced a strong $A B C$ phenomenon, with the $A B C_{\text {index }}$ reaching 0.25 , while $A B C_{\text {index }}$ of repeated $30 \mathrm{~mol} \% \mathrm{PE}$ injection was 0.7 . It was suggested that the reason for the weak $A B C$ phenomenon after the repeated injection of 30 $\mathrm{mol} \%$ PE may be that the higher density of PEG modification inhibited the production of anti-PEG antibodies. When the level of anti-PEG IgM reached a higher level, the $30 \mathrm{~mol} \%$ PE would still be cleared quickly with an accumulation in the liver and spleen (Fig. 5D).

The $A B C_{\text {index }}$ of the second injection of $30 \mathrm{~mol} \%$ PE-DSPG in the $A B C(+)$ rats reached 0.82 , which was slightly lower than the repeated injection of $30 \mathrm{~mol} \%$ PE-DSPG and further demonstrated the attenuation effect of DSPG on the ABC phenomenon; although there was a high level of anti-PEG IgM in rats, a strong $A B C$ phenomenon did not occur. The $A B C(+)$ rats injected with PE-DSPG-30 did not cause the $A B C$ phenomenon. This also showed that higher DSPG concentrations could effectively inhibit the occurrence of the $A B C$ phenomenon. 


\subsection{IgM binding of $30 \mathrm{~mol} \% \mathrm{PE}$ and $\mathbf{3 0} \mathrm{mol} \% \mathrm{PE}-\mathrm{DSPG}$ in the $\mathrm{ABC}(+)$ rats}

To determine the influence of DSPG on the intensity of the ABC phenomenon of PEs, we measured the changes in anti-PEG IgM before and $6 \mathrm{~h}$ after the second injection of $30 \mathrm{~mol} \% \mathrm{PE}, 30 \mathrm{~mol} \%$ PE-DSPG, and PE-DSPG-30 in the ABC (+) rats. As shown in Fig. 6, no significant change was observed in the plasma anti-PEG IgM levels of the ABC (+) rats $6 \mathrm{~h}$ after the injection of $30 \mathrm{~mol} \%$ PE-DSPG and PE-DSPG30 , while the level of anti-PEG IgM in the ABC $(+)$ rats decreased significantly after the intravenous injection of $30 \mathrm{~mol} \% \mathrm{PE}$, indicating that the second injection of $30 \mathrm{~mol} \% \mathrm{PEs}$ could be combined with anti-PEG IgM of the ABC (+) rats to produce the ABC phenomenon. In contrast, $30 \mathrm{~mol} \%$ PE-DSPG and PE-DSPG-30 combination did not enable the anti-PEG IgM recognition in the ABC (+) rats; therefore, the $A B C$ phenomenon did not occur.

\subsection{Co-injection of $10 \mathrm{~mol} \% \mathrm{PE}$ with $30 \mathrm{~mol} \% \mathrm{PE}, 30 \mathrm{~mol} \%$ PE-DSPG and DSPG-PE-30}

Here, we investigated the effect of the co-injection of $10 \mathrm{~mol} \% \mathrm{PE}$ containing DiR with blank $30 \mathrm{~mol} \% \mathrm{PE}$ or DSPG-PE-30 into the ABC (+) rats. As shown in Fig. 7, the co-injection of $30 \mathrm{~mol} \%$ PE and DSPG-PE-30 and $10 \mathrm{~mol} \% \mathrm{PE}$ did not affect the $\mathrm{ABC}$ phenomenon of $10 \mathrm{~mol} \% \mathrm{PE}$.

\section{Discussion}

The induction of the immune reaction by the first dose of PEGylated nanocarriers, eliminating the longcycle characteristics of the second-dose PEGylated nanocarriers, is called the ABC phenomenon. Many studies have reported that the main reason for the occurrence of the $A B C$ phenomenon is the recognition of the PEGylated nanocarriers of the secondary injection by the anti-PEG IgM leading to accelerated clearance of the second dose of PEGylated nanocarriers. In previous reports, it was confirmed that the binding site between PEG and the hydrophobic segment of PEG-lipid derivatives such as $\mathrm{mPEG} 2000^{-} \mathrm{DSPE}$ was the main binding site recognized by anti-PEG IgM. The findings of a previous study [30] showed that recognition binding could be eliminated by adding a hydrophilic segment between the PEG and the hydrophobic segment. Therefore, masking the binding site between PEG and the hydrophobic segment of mPEG-DSPE may weaken or eliminate the ABC phenomenon. Here, by controlling the particle size of the emulsions, excluding the influence of the release behavior, and only investigating the influence of the addition of DSPG on ABC phenomenon,we further proved the importance of the interface confrontation between PEG and the hydrophobic segment, recognized by anti-PEG IgM to induce the ABC phenomenon.

DSPG is an anionic phospholipid with the same saturated fat chain as MPEG-DSPE. The addition of DSPG to PEs weakened the ABC phenomenon significantly; however, the antibody-induced ability of the APA response did not decrease, and it still induced the production of anti-PEG IgM. Using cross-injections and co-injections, we confirmed that the recognition and binding abilities of anti-PEG IgM to PEs 
containing DSPG was weakened significantly, and that the $A B C$ phenomenon disappeared when the molar ratio of DSPG to $\mathrm{mPEG}_{2000}$-DSPE was 7:3 and 30:1. In comparing the structures of DSPG and SPC

(Fig. 8), it was found that the two fatty chains of SPC were unsaturated and could not form a neat emulsion layer with the two saturated fat chains of MPEG-DSPE; however, DSPG had the same fatty chain as DSPE, and the two could form a more neat and uniform emulsion layer. From the perspective of spatial structure, the two free hydroxyl groups of DSPG being adjacent to the amide bond in MPEG2000DSPE, may, to some extent, mask the amide bond.

\section{Conclusions}

In this study, in order to weaken or eliminate the ABC phenomenon, we investigated the ability of DSPG to mask the recognition sites of anti-PEG IgM to PEs. We verified the potential mechanisms of the ABC phenomenon. By using co-injections or cross-injections with common PEs, the ability of PEs containing DSPG to bind to IgM was significantly weakened. When the content of DSPG reached a specific value, the $A B C$ phenomenon disappeared and did not change the titer of anti-PEG IgM in the plasma of the ABC (+) rats. Therefore, this study further demonstrated that the hydrophobic interface between the PEG and the hydrophobic segment is an important site for the recognition and binding of anti-PEG IgM, and thus, by masking this site, the $A B C$ phenomenon can be effectively reduced.

\section{Declarations}

\section{Ethical statement}

\section{Ethics approval and consent to participate}

All authors agreed with the content and that all gave explicit consent to submit and that they obtained consent from the responsible authorities at the institute/organization where the work has been carried out, before the work is submitted.

\section{Consent for publication}

Informed consent was obtained from all individual participants included in the study

\section{Availability of data and material}

All data and material are available.

\section{Competing interests}

The authors declare that they have no known competing financial interests or personal relationships that could have appeared to influence the work reported in this paper.

\section{Funding}


This work was supported by the National Natural Science Foundation of China (No. 81973271).

\section{Authors' contributions}

All authors contributed to the study conception and design. Material preparation, data collection and analysis were performed by Zhe Li, Xin Gao and Xinyang Yan. The first draft of the manuscript was written by Zhe Li and all authors commented on previous versions of the manuscript. All authors read and approved the final manuscript.

\section{Compliance with ethical standards}

All institutional and national guidelines for the care and use of laboratory animals were followed.

\section{References}

1. D'Souza AA, Shegokar R. Polyethylene glycol (PEG): a versatile polymer for pharmaceutical applications. Expert Opin Drug Deliv. 2016;13(9):1257-75. https://doi.org/10.1080/17425247.2016.1182485.

2. Torchilin VP. Polymer-coated long-circulating microparticulate pharmaceuticals. J Microencapsul. 1998;15(1):1-19. https://doi.org/10.3109/02652049809006831.

3. Abuchowski A, van Es TV, Palczuk NC, Davis FF. Alteration of immunological properties of bovine serum albumin by covalent attachment of polyethylene glycol. J Biol Chem. 1977;252(11):3578-81.

4. Abuchowski A, McCoy JR, Palczuk NC, van Es TV, Davis FF. Effect of covalent attachment of polyethylene glycol on immunogenicity and circulating life of bovine liver catalase. J Biol Chem. 1977;252(11):3582-6.

5. Roberts MJ, Bentley MD, Harris JM. Chemistry for peptide and protein pegylation. Adv Drug Deliv Rev. 2002;54(4):459-76. https://doi.org/10.1016/s0169-409x(02)00022-4.

6. Armstrong JK, Hempel G, Koling S, Chan LS, Fisher T, Meiselman HJ, et al. Antibody against poly(ethylene glycol) adversely affects PEG-asparaginase therapy in acute lymphoblastic leukemia patients. Cancer. 2007;110(1):103-11. https://doi.org/10.1002/cncr.22739.

7. Hershfield MS, Ganson NJ, Kelly SJ, Scarlett EL, Jaggers DA, Sundy JS. Induced and pre-existing anti-polyethylene glycol antibody in a trial of every 3-week dosing of pegloticase for refractory gout, including in organ transplant recipients. Arthritis Res Ther. 2014;16(2):R63. https://doi.org/10.1186/ar4500.

8. Mohamed M, Abu Lila AS, Shimizu T, Alaaeldin E, Hussein A, Sarhan HA, et al. Pegylated liposomes: immunological responses. Sci Technol Adv Mater. 2019;20(1):710-24. https://doi.org/10.1080/14686996.2019.1627174.

9. Liu Y, Smith CA, Panetta JC, Yang W, Thompson LE, Counts JP, et al. Antibodies predict pegaspargase allergic reactions and failure of rechallenge. J Clin Oncol. 2019;37(23):2051-61. 
10. Dams ET, Laverman P, Oyen WJ, Storm G, Scherphof GL, van Der Meer JW, Corstens FH, Boerman OC. Accelerated blood clearance and altered biodistribution of repeated injections of sterically stabilized liposomes. J Pharmacol Exp Ther. 2000;292(3):1071-9.

11. Mima Y, Hashimoto Y, Shimizu T, Kiwada H, Ishida T. Anti-PEG IgM is a major contributor to the accelerated blood clearance of polyethylene glycol-conjugated protein. Mol Pharm. 2015;12(7):2429-35.

12. Su Y, Liu M, Xiong Y, Ding J, Liu X, Song Y, et al. Effects of stability of pegylated micelles on the accelerated blood clearance phenomenon. Drug Deliv Transl Res. 2019;9(1):66-75.

13. Hara E, Ueda M, Makino A, Hara I, Ozeki E, Kimura S. Factors Influencing in vivo disposition of polymeric micelles on multiple administrations. ACS Med Chem Lett. 2014;5(8):873-7.

14. Liu M, Chu Y, Liu H, Su Y, Zhang Q, Jiao J, et ai. Accelerated Blood Clearance of Nanoemulsions Modified with PEG-Cholesterol and PEG-Phospholipid Derivatives in Rats: The Effect of PEG-Lipid Linkages and PEG Molecular Weights. Mol Pharm. 2020;17(4):1059-70.

15. Joshi M, Pathak S, Sharma S, Patravale V. Solid microemulsion preconcentrate (NanOsorb) of artemether for effective treatment of malaria. Int J Pharm. 2008;362(1-2):172-8.

16. Lu W, Wan J, She Z, Jiang X. Brain delivery property and accelerated blood clearance of cationic albumin conjugated pegylated nanoparticle. J Control Release. 2007;118(1):38-53.

17. Zhang P, Sun F, Liu S, Jiang S. Anti-PEG antibodies in the clinic: current issues and beyond pegylation. J Control Release. 2016;244(B):184-93.

18. Ganson NJ, Kelly SJ, Scarlett E, Sundy JS, Hershfield MS. Control of hyperuricemia in subjects with refractory gout, and induction of antibody against poly (ethylene glycol) (PEG), in a phase I trial of subcutaneous pegylated urate oxidase. Arthritis Res Ther. 2006;8(1):R12.

19. Lipsky PE, Calabrese LH, Kavanaugh A, Sundy JS, Wright D, Wolfson M, et al. Pegloticase immunogenicity: the relationship between efficacy and antibody development in patients treated for refractory chronic gout. Arthritis Res Ther. 2014;16(2):R60.

20. Rau RE, Dreyer ZA, Choi MR, Liang W, Skowronski R, Allamneni KP, et al. Outcome of pediatric patients with acute lymphoblastic leukemia/lymphoblastic lymphoma with hypersensitivity to pegaspargase treated with pegylated Erwinia asparaginase, pegcrisantaspase: A report from the Children's Oncology Group. Pediatr Blood Cancer. 2018;65(3):e26873.

21. Park K. Attenuating the immunogenicity of PEGylated liposomes by gangliosides. Journal of controlled release: official journal of the Controlled Release Society. 2017;250:116. https://doi.org/10.1016/j.jconrel.2017.03.002.

22. Zhang T, Zhou S, Kang L, Luo X, Liu Y, Song Y, et al. The effect of monosialylganglioside mix modifying the pegylated liposomal epirubicin on the accelerated blood clearance phenomenon. Asian J Pharm Sci. 2017;12(2):134-42. https://doi.org/10.1016/j.ajps.2016.06.005.

23. Mcsweeney MD, Price LSL, Wessler T, Ciociola EC, Herity LB, Piscitelli JA, et al. Overcoming anti-PEG antibody mediated accelerated blood clearance of pegylated liposomes by pre-infusion with high molecular weight free PEG. J Control Release. 2019;311-312(21):138-46. 
24. Koide H, Asai T, Hatanaka K, Urakami T, Ishii T, Kenjo E, et al. Particle size-dependent triggering of accelerated blood clearance phenomenon. Int J Pharm. 2008;362(1-2):197-200.

25. Bavli Y, Winkler I, Chen BM, Roffler S, Cohen R, Szebeni J, et al. Doxebo (doxorubicin-free Doxil-like liposomes) is safe to use as a pre-treatment to prevent infusion reactions to pegylated nanodrugs. $J$ Control Release. 2019;306(Suppl 4):138-48.

26. Li CL, Zhao X, Wang YJ, Yang HY, Li HX, Li H, et al. Prolongation of time interval between doses could eliminate accelerated blood clearance phenomenon induced by pegylated liposomal topotecan. Int $\mathrm{J}$ Pharm. 2013;443(1-2):17-25.

27. Zhang T, Zhou S, Kang L, Luo X, Liu Y, Song Y, et al. The effect of monosialylganglioside mix modifying the pegylated liposomal epirubicin on the accelerated blood clearance phenomenon. Asian J Pharm Sci. 2017;12(2):134-42.

28. Ohmae M, Kojima M, Mihara K, Yamazaki Y, Hara I, Hara E, et al. Reduced immune response to polymeric micelles coating sialic acids. Bioorg Med Chem Lett. 2016;26(20):4976-82.

29. Abu Lila AS, Uehara Y, Ishida T, Kiwada H. Application of polyglycerol coating to plasmid DNA lipoplex for the evasion of the accelerated blood clearance phenomenon in nucleic acid delivery. $J$ Pharm Sci. 2014;103(2):557-66.

30. Shiraishi K, Hamano M, Ma H, Kawano K, Maitani Y, Aoshi T, et al. Hydrophobic blocks of PEGconjugates play a significant role in the accelerated blood clearance (ABC) phenomenon. J Control Release. 2013;165(3):183-90.

\section{Figures}



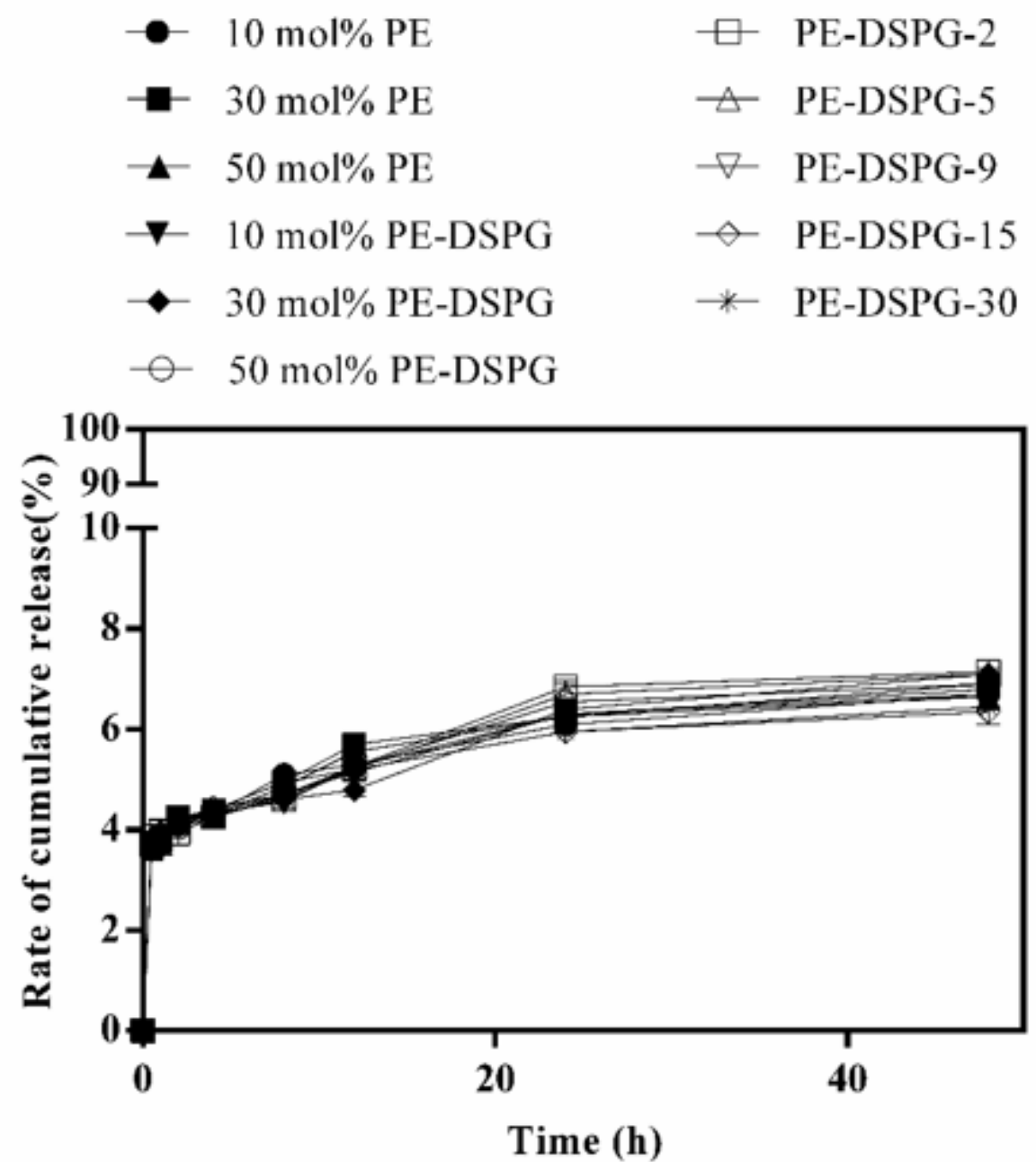

Figure 1

The rate of the DiR cumulative releases from PEs in $48 \mathrm{~h}$ in vitro. The release kinetics follows RitgerPeppas equation. Data are shown as means \pm standard, $n=3$. 


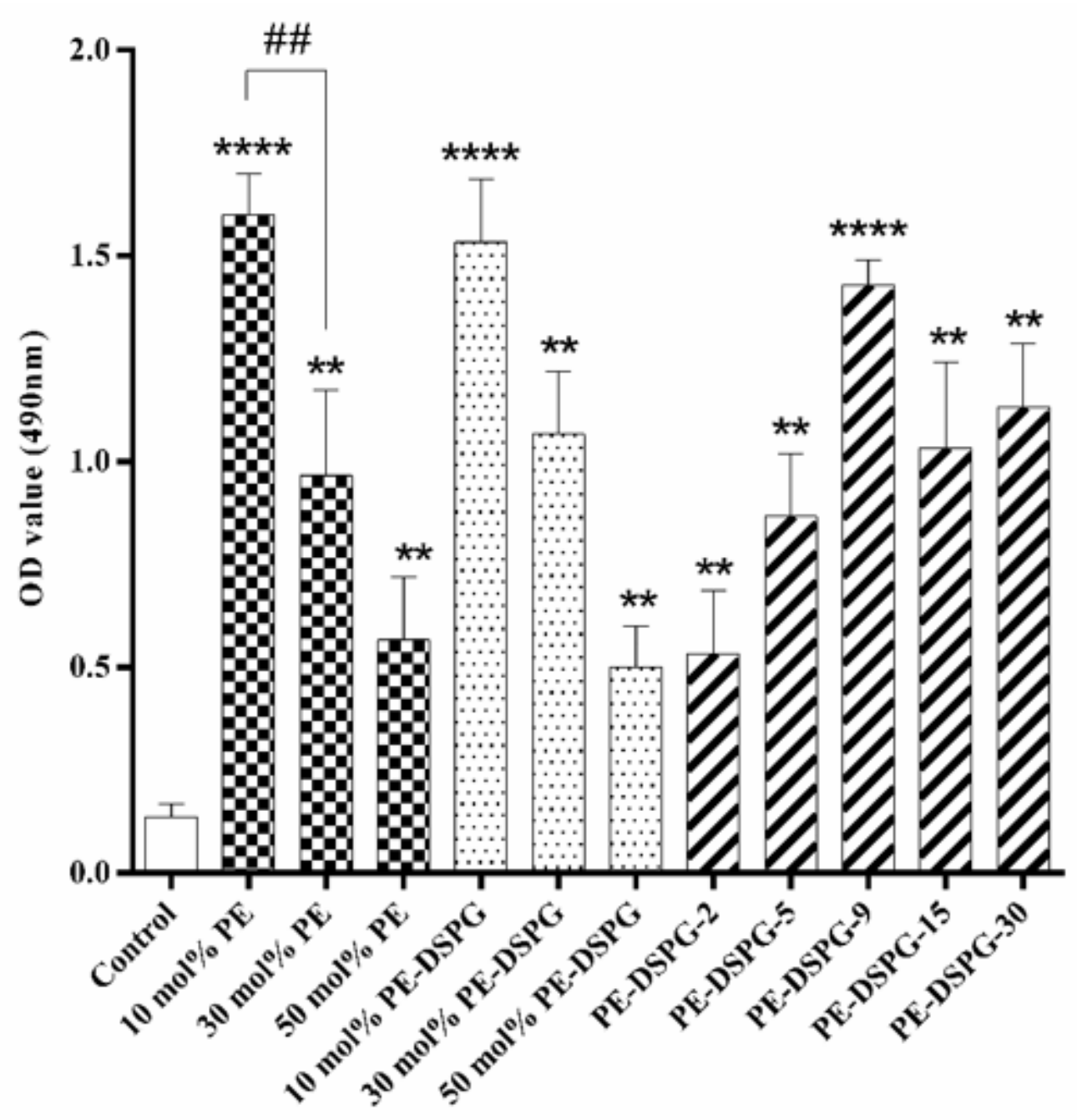

Figure 2

Determination of anti-PEG IgM titer in rats seven days after the first dose of PEs. Significant difference compared with control is indicated by *, and \# means significant difference between groups. Data are shown as means $\pm S D s, n=3$. ${ }^{\star} P<0.05$, ${ }^{\star} P<<0.01$, $* \star * P<0.001$, $* \star \star * P<0.0001$. 
A
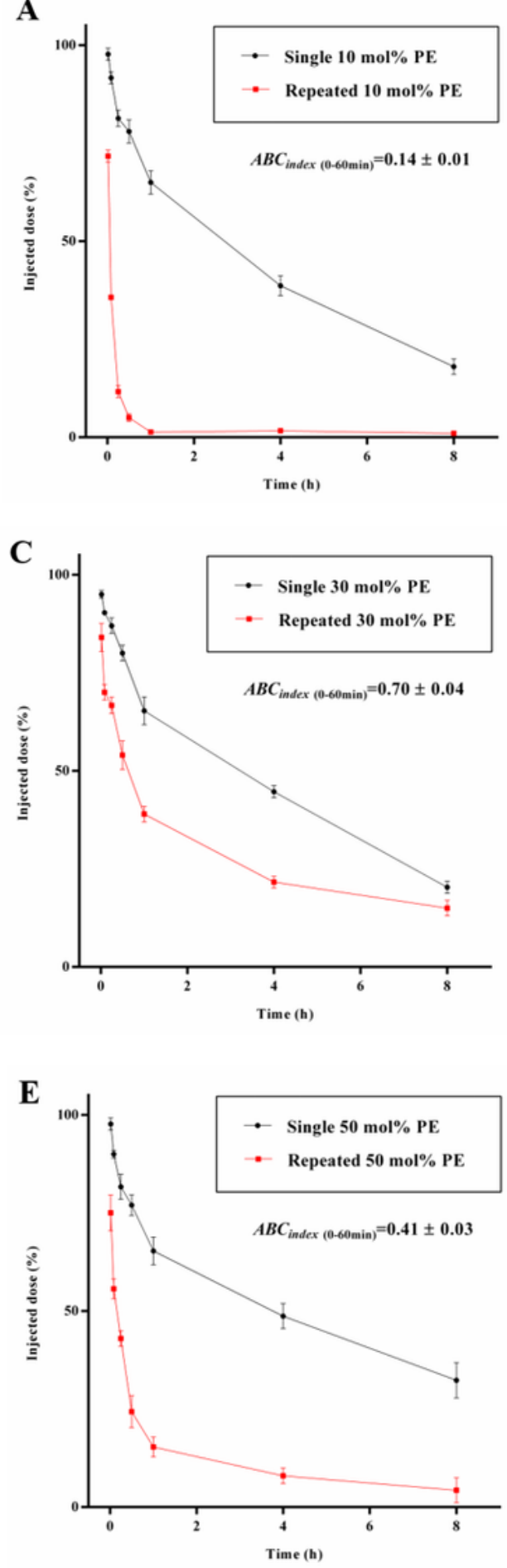

B
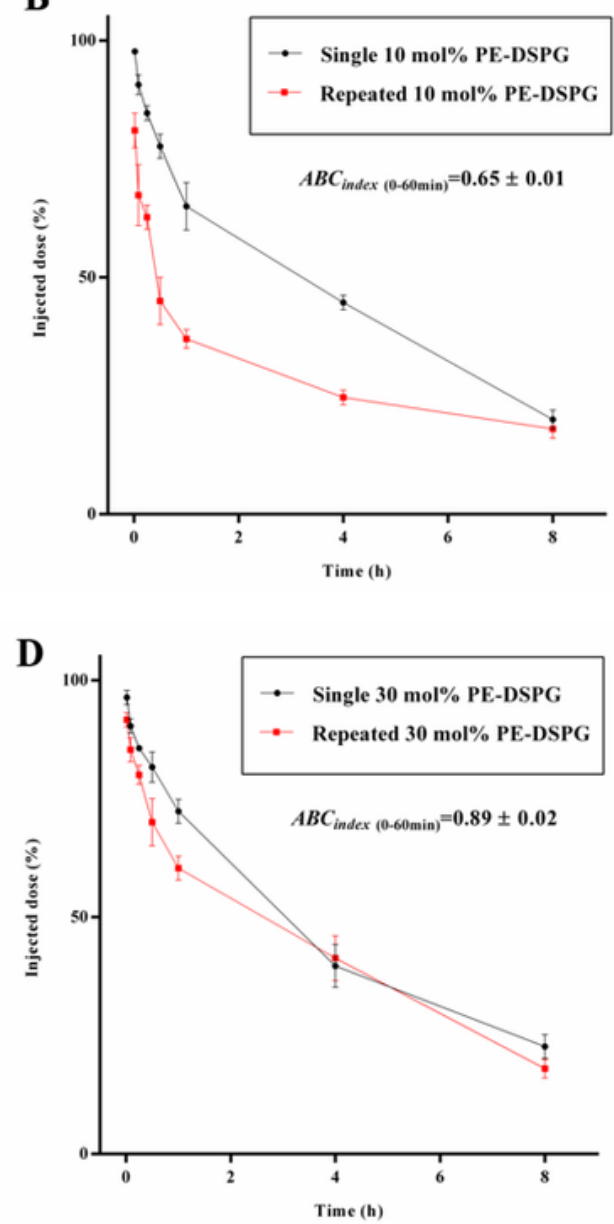

F

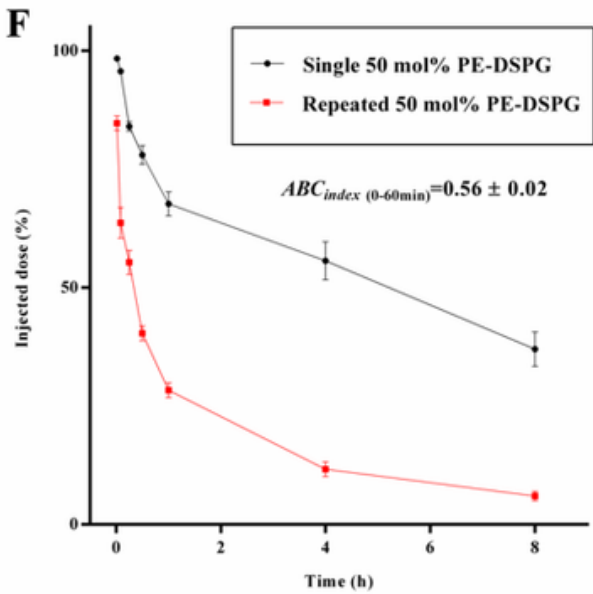

\section{Figure 3}

Blood clearance profile of single (black) or repeated (red) injections with an interval of seven days of $n$ mol \% PEs and PE-DSPGs loading DiR with different densities of PEG in rats ( $n=10$ [A and B], 30 [C and D], 50 [E and F]). Data are shown as means \pm SDs, $n=3$. 
A

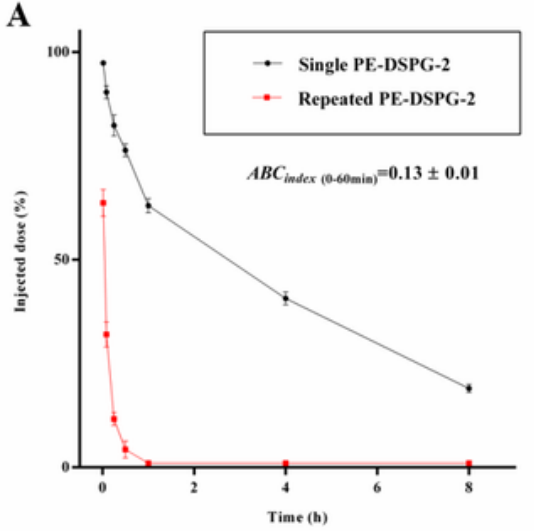

C

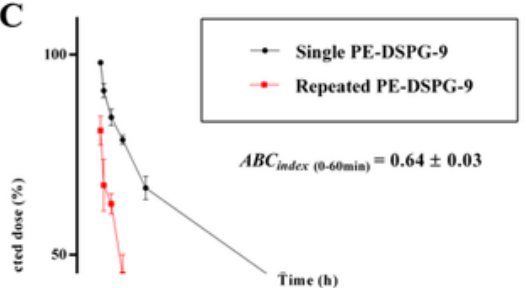

E

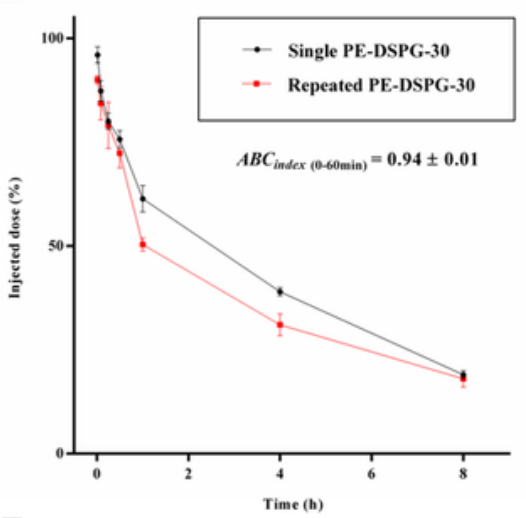

E

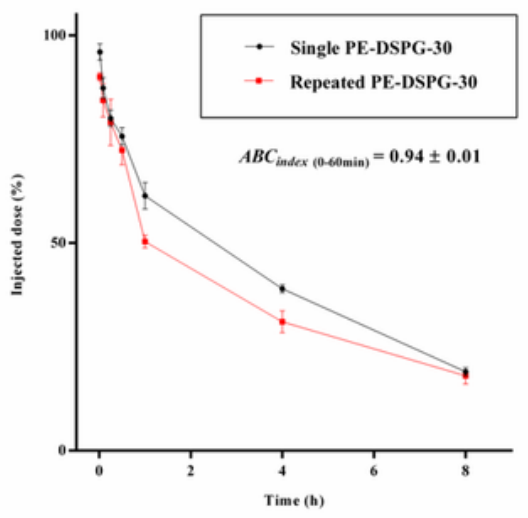

B

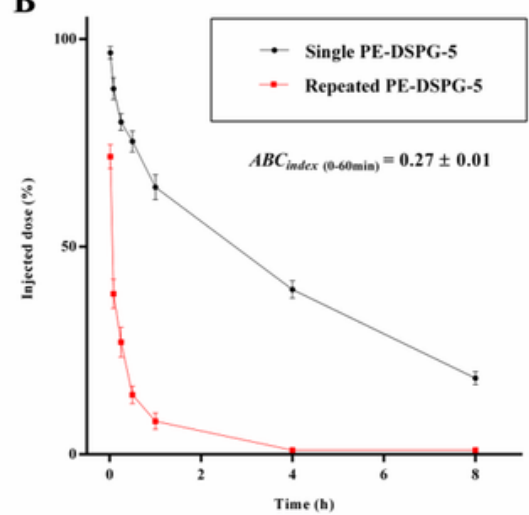

D

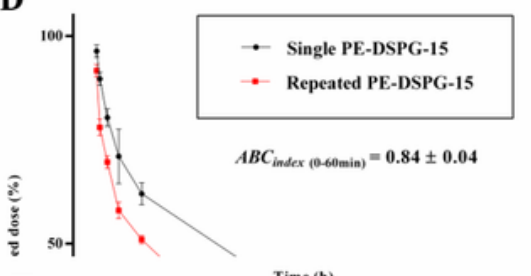

F

Time (h)

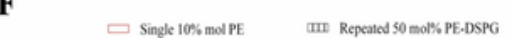

$\neg$ Repeased 10 mols; PE

$\square$ Repeasid 10 molk PE PE

Single $30 \%$ mol PE
Repeated 30 mols PE

- Single $50 \%$ mol PE all Repeuted PE-DSPG-5

$=0$ Repeated 50 molk PE $\quad$ and Single PE.DSPG.9

Ww Single $10 \%$ mol PE-DSPG ans Repested PE.DSPG.9

W Repented 10 mollo PE-DSPG W Single PE-DSPG-15

- Single 30N mol PE.DSPG W Repeated PE.DSPG-15

- Reprated 30 molls PE-DSPG $\mathbf{Z}$ Single PE.DSPG-30

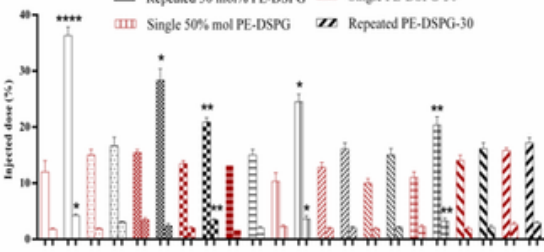

F

Time (h)

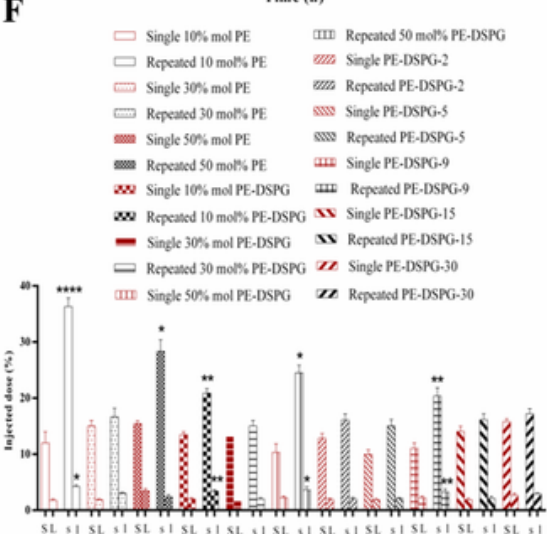

Figure 4

Blood clearance profile of single (black) or repeated (red) injections with an interval of seven days of PEDSPG-ns [n = 2 (A), 5 (B), 9 (C), 15 (D), 30 (E)] loading DiR with different concentration of DSPG in rats. (F) Accumulation of liver and spleen $8 \mathrm{~h}$ after single (red) and repeated (black) injection of DiR-PEs in rats. $S$ and $L$ represents accumulation in liver and spleen after single injection; $S$ and I represent accumulation in liver and spleen after repeated injection. Significant differences between the 
accumulation of single and repeated injection of the same PEs in the same tissue is indicated by **P $<$ $0.05, * * \mathrm{P}<0.01, * \star \star * \mathrm{P}<0.0001$. Data are shown as means $\pm \mathrm{SD}, \mathrm{n}=3$.
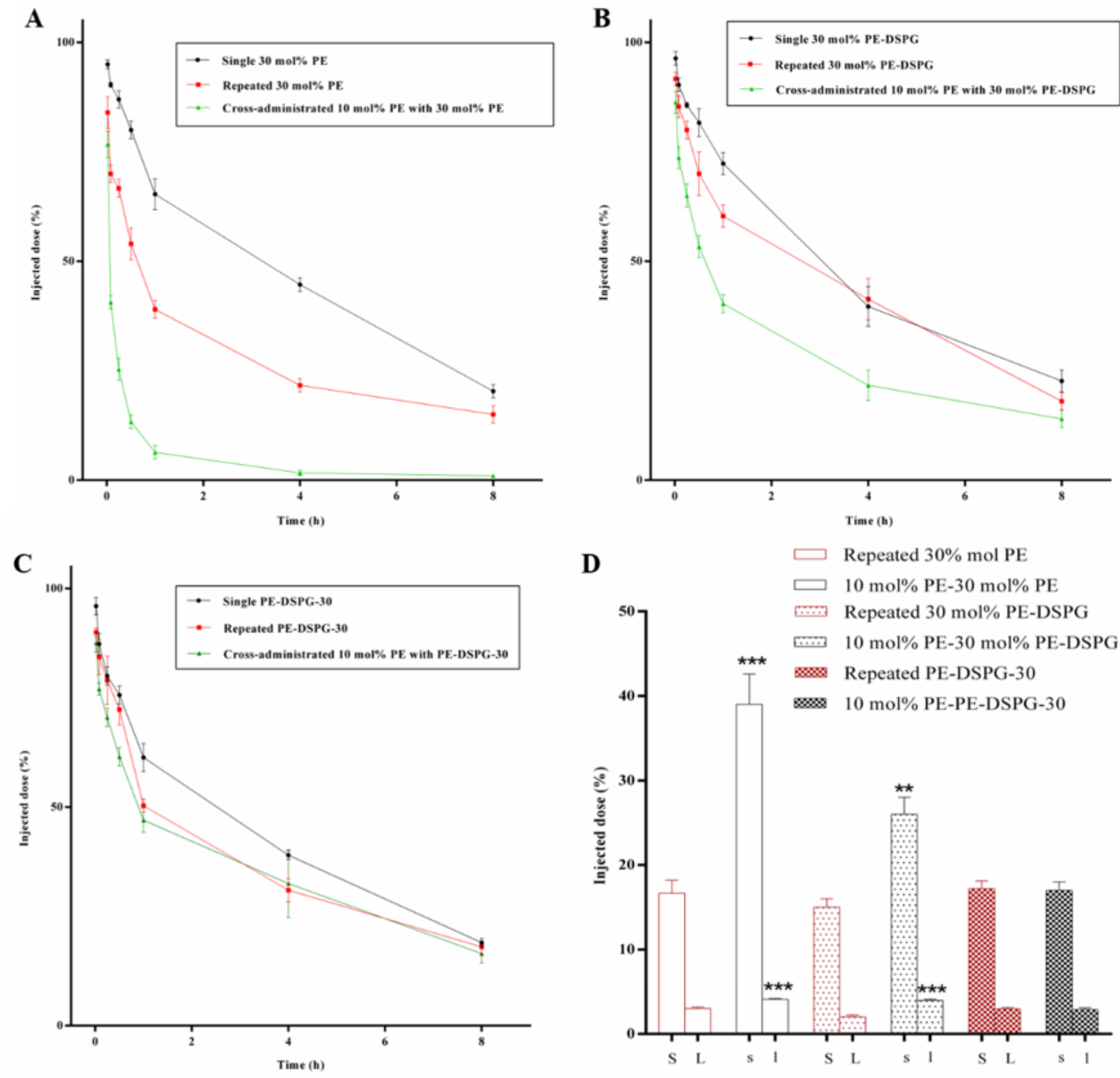

Figure 5

Blood clearance profiles of cross-injection (green) of $10 \mathrm{~mol} \% \mathrm{PE}$ as the first dose with $30 \mathrm{~mol} \% \mathrm{PE}(\mathrm{A})$, $30 \mathrm{~mol} \%$ PE-DSPG (B), and PE-DSPG-30 (C) loading DiR as the second dose (repeated injection of same PEs, red). (D) Accumulation in liver and spleen $8 \mathrm{~h}$ after single (red) and cross (black) injection of DiR-PEs in rats. $S$ and $L$ represents the accumulation in liver and spleen after repeated injection; $S$ and I represent accumulation in liver and spleen after cross-injection. Significant differences between the accumulation 
of repeated and cross injection of the same PEs in the same tissue is indicated by *. ${ }^{*} P<0.05, * * P<0.01$, $\star * * P<0.001$. Data are shown as means $\pm S D s, n=3$.

- Single PE injection

6h after repeated PE injection

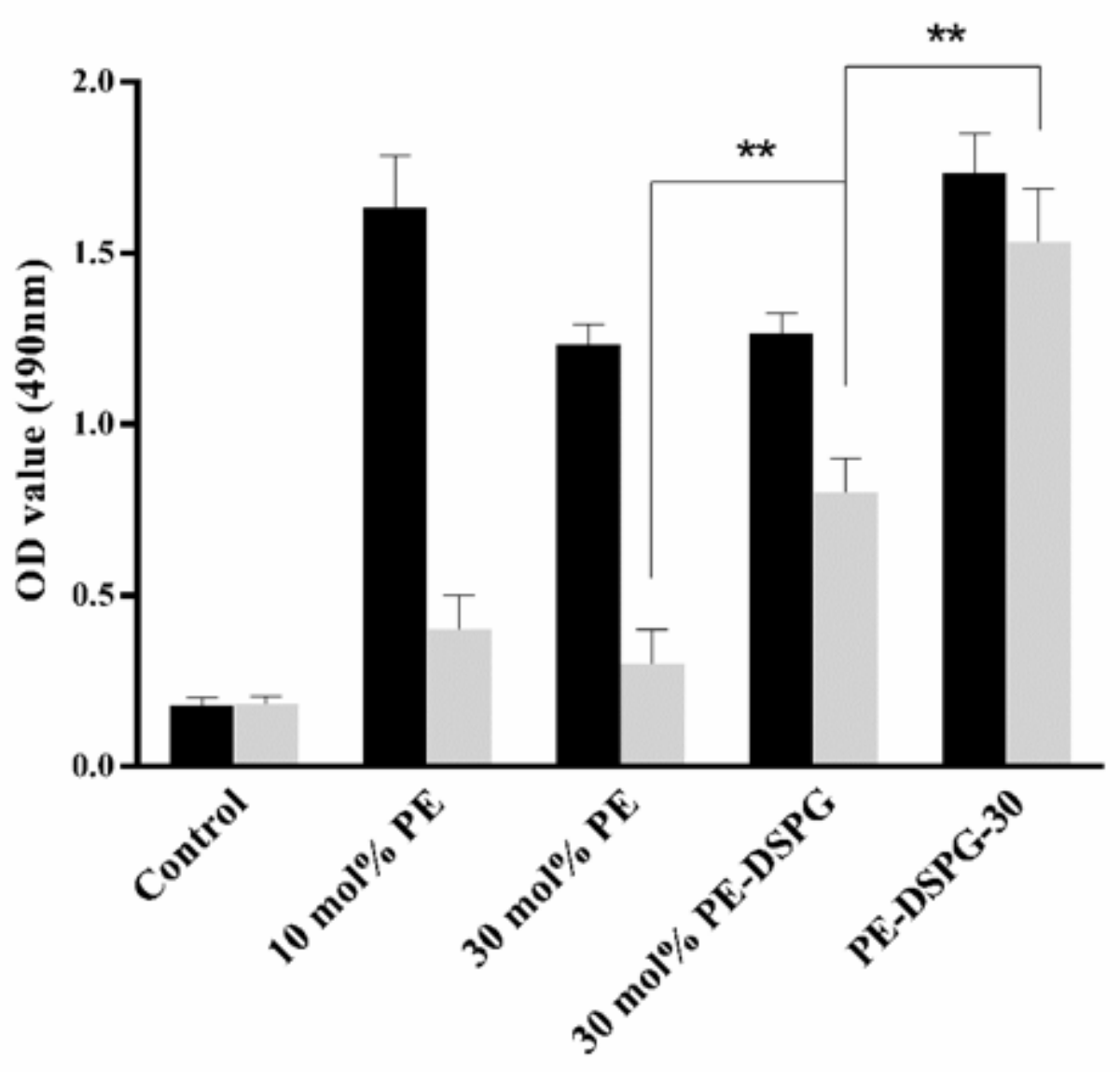

Figure 6

Changes of anti-PEG IgM titer 7 days after the first dose (black) and $6 \mathrm{~h}$ after the second dose (gray) of $10 \mathrm{~mol} \%$ PE, $30 \mathrm{~mol} \%$ PE, $30 \mathrm{~mol} \%$ PE-DSPG and PE-DSPG-30. Significant differences between the titer are indicated by $*{ }^{*} \mathrm{P}<0.01$, Data are shown as means $\pm S D s, n=3$. 

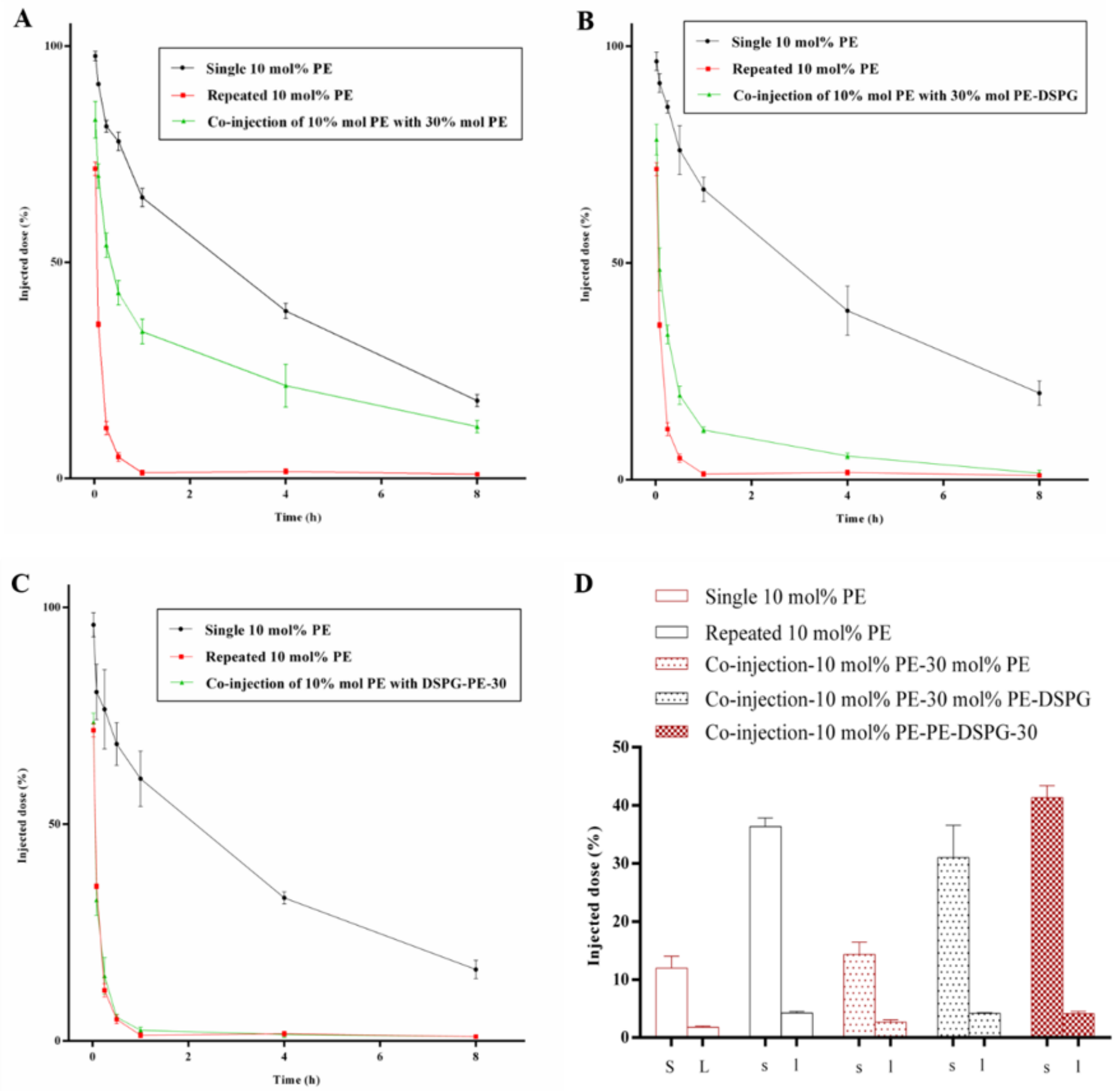

\section{Figure 7}

Blood clearance profiles of co-injection (green) of $30 \mathrm{~mol} \%$ PEs (A), $30 \mathrm{~mol} \%$ PE-DSPGs (B), and PEDSPG-30s (C) with $10 \mathrm{~mol} \%$ PEs loading DiR as the second dose (repeated same PEs, red). (D) Accumulation in liver and spleen $8 \mathrm{~h}$ after single $10 \mathrm{~mol} \% \mathrm{PE}$ injection, repeated $10 \mathrm{~mol} \% \mathrm{PE}$ injection and co-injection of DiR-PEs in rats. S and L represents accumulation of liver and spleen after repeated injection; $s$ and I represent accumulation of liver and spleen after repeated dose and co-injection. Data are shown as means $\pm \mathrm{SDs}, \mathrm{n}=3$. 
<smiles>CCCCC/C=C\C/C=C\CCCCCCCCCCCCC(=O)OC[C@H](O)COP(=O)([O-])OCC[N+](C)(C)C</smiles><smiles>CCCCCCCCCCCCCCCCCC(=O)OC[C@@H](COP(=O)([O-])OCC(O)CO)OC(=O)CCCCCCCCCCCC</smiles><smiles>[2H]C([2H])(COC(=O)COC(=O)CCCCCCCCCCCCCCCCCCCCCCCCC)OP(=O)([O-])OCCNC(=O)OCCOC</smiles>

Figure 8

Structure of SPC (A), DSPG (B), and mPEG2000-DSPE (C).

\section{Supplementary Files}

This is a list of supplementary files associated with this preprint. Click to download.

- Scheme1.png

- SupplementaryMaterial.docx 